\title{
Prognostic significance of preexisting interstitial lung disease in Japanese patients with small-cell lung cancer.
}

\section{$\operatorname{AUTHOR}(\mathrm{S})$ :}

Togashi, Yosuke; Masago, Katsuhiro; Handa, Tomohiro; Tanizawa, Kiminobu; Okuda, Chiyuki; Sakamori, Yuichi; Nagai, Hiroki; Kim, Young Hak; Mishima, Michiaki

\section{CITATION:}

Togashi, Yosuke ... [et al]. Prognostic significance of preexisting interstitial lung disease in Japanese patients with small-cell lung cancer.. Clinical lung cancer 2012, 13(4): 304-311

\section{ISSUE DATE:}

2012-07

URL:

http://hdl.handle.net/2433/158292

\section{RIGHT:}

(c) 2012 Elsevier Inc.; この論文は出版社版でありません。引用の際には 出版社版をご確認ご利用ください。; This is not the published version. Please cite only the published version. 
Title:

Prognostic Significance of Preexisting Interstitial Lung Disease in Japanese Patients with Small Cell Lung Cancer

Author's name:

Yosuke Togashi, Katsuhiro Masago, Tomohiro Handa, Kiminobu Tanizawa, Chiyuki Okuda, Yuichi Sakamori, Hiroki Nagai, Young Hak Kim, Michiaki Mishima

\section{Author's Affiliation:}

Department of Respiratory Medicine, Graduate School of Medicine, Kyoto University, Kyoto, Japan

\section{Grant support:}

Nothing

\section{Running title:}

Prognosis in SCLC patients with ILD

\section{Corresponding author:}

Yosuke Togashi, M.D.

Department of Respiratory Medicine, Graduate School of Medicine, Kyoto University, 54 Shogoin-Kawaharacho, Sakyo-ku, Kyoto 606-8507 Japan.

Phone: +81-75-751-3830; Fax: +81-75-751-4643

E-mail: ytogashi@kuhp.kyoto-u.ac.jp.

\section{Number of words:}

Abstract: 246 words, Manuscript: 2172 words

\section{Number of characters:}

Abstract: 1403 characters, Manuscript: 12234 characters 


\section{Conflict of interest statement}

All authors have no conflicts of interest. 


\section{MicroAbstract}

We retrospectively investigated patients with small-cell lung cancer with or without interstitial lung disease (ILD). Response rates and median progression-free survival of 1st-line chemotherapy in patients with or without preexisting ILD was not significantly different. However, pneumonitis associated with chemotherapy was significantly increased in patients with preexisting ILD, and preexisting ILD is an independent prognostic factor for poorer survival. 


\begin{abstract}
Background: In Japan, iatrogenic acute exacerbation (AE) of interstitial lung disease (ILD) is a serious complication in patients with lung cancer and simultaneous ILD. Some reports suggest that patients with ILD and small cell lung cancer (SCLC) might benefit from chemotherapy, but the influence of ILD on prognosis is unclear. Patients and Methods: Retrospective study of patients with SCLC with or without ILD. Between April 2006 and March 2011, 122 SCLC patients receiving platinum-based combination chemotherapy participated. Results: Twenty-eight patients (23.0\%) had ILD at diagnosis. Pneumonitis associated with chemotherapy including AE-ILD was significantly increased in patients with preexisting ILD $(P=.0001 ; 8 / 28$ vs. $2 / 94$, $)$. In patients receiving chemotherapy alone, response rates and median progression-free survival of 1st-line chemotherapy in patients with or without preexisting ILD was not significantly different $(P=.26 ; 20 / 26$ vs. 52/60 and $P=.089 ; 4.4$ months vs. 5.4 months, respectively). Median overall survival (OS) of all patients was 15.5 months, but those without preexisting ILD survived significantly longer $(P=.0010 ; 17.8$ months vs. 10.7 months). Multivariate analysis revealed that performance status of 0 or 1 (hazard ratio $[\mathrm{HR}], 0.19 ; 95 \%$ confidence interval $[\mathrm{CI}], 0.10-0.37 ; P<.0001)$, limited disease (HR, 0.42; $95 \% \mathrm{CI}, 0.23-0.73 ; P=.0017)$, and no preexisting ILD (HR, 0.36; 95\% CI, $0.19-0.69 ; P=.0027$ ) were significantly associated with longer OS. Conclusion: Patients with SCLC and ILD might benefit from chemotherapy but preexisting ILD is an independent prognostic factor for poorer survival.
\end{abstract}

Key words: Small cell lung cancer, interstitial lung disease, acute exacerbation, idiopathic pulmonary fibrosis, chemotherapy 


\section{Introduction}

Lung cancer remains the leading cause of cancer-related death in Japan as well as in Western countries. Although the incidence of small cell lung cancer (SCLC) compared to other lung cancer is relatively low (approximately 15\% of all), prognosis remains poor, even for early stage SCLC. From the time of diagnosis, median survival for limited disease (LD)-SCLC and extensive disease (ED)-SCLC is $15-20$ and 8-13 months, respectively. ${ }^{1-3}$

Interstitial lung disease (ILD) is characterized by fibrosis of the interstitial compartment clinically manifesting as dyspnea. ILD, especially idiopathic pulmonary fibrosis (IPF), seems to be associated with lung carcinogenesis. The incidence of lung cancer in patients with IPF is higher than in the general population. ${ }^{4-7}$ ILD is usually characterized by slowly progressive respiratory insufficiency. However, the clinical course of ILD is often complicated by acute deterioration without an identifiable cause, called "acute exacerbation" (AE-ILD), and these processes are associated with poor prognoses. ${ }^{8-11}$ Moreover, in patients with lung cancer together with ILD, AE-ILD frequently occurs after anticancer therapy including chemotherapy. However, there are few reports on chemotherapy for lung cancer with ILD. ${ }^{12-15}$ If left untreated, patients with SCLC rarely survive longer than a few months, but $60-80 \%$ respond to chemotherapy, which dramatically prolongs survival compared to the best supportive care. ${ }^{3}$ Although Minegishi et al. suggested that patients with SCLC and ILD might benefit from chemotherapy. ${ }^{12,14}$ the influence of ILD on prognosis has not been reported. In the present article, we retrospectively compared patients with SCLC with or without ILD. 


\section{Materials and methods}

\section{Patients}

One hundred and forty-one Japanese patients with untreated SCLC were diagnosed between April 2006 and March 2011 at Kyoto University Hospital. Of these, 19 received best supportive care alone, and the remaining 122 received platinum-based combination chemotherapy (platinum plus etoposide or irinotecan) as 1st-line chemotherapy. These latter 122 patients participated in this study, which was approved by the Ethics Committee of Kyoto University Graduate School and Faculty of Medicine.

\section{Patients' characteristics and tumor responses}

We retrospectively analyzed patients' backgrounds and tumor responses. Staging was performed based on the Verterans Administration Lung Study Group system, ${ }^{1}$ and responses were assessed every 2 cycles of chemotherapy according to the Response Evaluation Criteria in Solid Tumor version 1.0. ${ }^{16}$ First-line chemotherapy was performed for up to 6 cycles at the discretion of the attending physician.

\section{Preexisting interstitial lung disease}

We classified preexisting ILD into two types, either idiopathic interstitial pneumonia (IIP) or not IIP. The latter included ILD associated with collagen-vascular disease (CVD), sarcoidosis, and other syndromes. IIP itself was classified into two types, IPF and non-IPF. For the diagnosis of IIP, we used the American Thoracic Society/European Respiratory Society consensus classification. ${ }^{7}$ None of the patients was surgically biopsied; thus, ILD was diagnosed by 2 physicians based on high-resolution computed tomography (HRCT) and clinical features before treatment for SCLC. Five patients underwent bronchoscopy (bronchoalveolar lavage and transbronchial lung biopsy) for more detailed examination of ILD. Pulmonary function test data in 91 patients $(74.6 \%)$ were available, and these are referred to here.

\section{Acute exacerbation of interstitial lung disease and pneumonitis}

AE-ILD was diagnosed according to published criteria: ${ }^{14,15,17}$ (a) subjective worsening of dyspnea within the last month; (b) new ground-glass opacities or consolidation on chest radiography or $\mathrm{HRCT}$; (c) hypoxemia with a decline of $10 \mathrm{mmHg}$ in $\mathrm{PaO}_{2}$ from the previous level; (d) no evidence of infection (assessed by negative respiratory culture and serologic test results for respiratory pathogens); and (e) no clinical evidence of pulmonary embolism, congestive heart failure, or pneumothorax as a cause of acute worsening. For patients without preexisting ILD, pneumonitis is considered equivalent to AE-ILD. Therefore, the grade was assessed as pneumonitis according to the Common Terminology Criteria for Adverse Events version 3.0 (CTCAE v3.0). 


\section{Statistical analysis}

The univariate relationship between each independent variable was examined using the $\chi^{2}$ test or Fisher's exact test if there were five or fewer observations in a group. Overall survival (OS) was defined as the time from the initiation of 1st-line chemotherapy until death from any cause. Progression-free survival (PFS) was defined as the time from the initiation of chemotherapy to the first observation of disease progression or death from any cause. OS and PFS were analyzed using the Kaplan-Meier method and compared among groups by the log-rank test. To identify prognostic factors, a Cox proportional hazards model was used for univariate and multivariate analyses. The multivariate analysis was performed with adjustment for covariates, including age, gender, smoking status, Eastern Cooperative Oncology Group Performance Status (ECOG PS), stage, and preexisting ILD. All tests were two-tailed and $P$ values less than 0.05 were considered statistically significant. All preceding statistical analyses were performed using JMP 8 software (SAS Institute, Cary, NC, USA). 


\section{Results}

The clinical characteristics of 122 patients are summarized in Table 1. All patients were Japanese, including 94 males (77.0\%) and 28 females (23.0\%), with a median age of 69 years (range, $33-85$ years). Many patients $(95 / 122,77.9 \%)$ were heavy smokers ( $>40$ pack-years) and had ECOG PS of 0 or $1(99 / 122,81.1 \%)$. The primary stage of 53 patients $(43.4 \%)$ was LD, with 69 (56.6\%) ED. Twenty-eight patients (23.0\%) had ILD at SCLC diagnosis and 9 of them (32.1\%) had already been followed by their physicians as ILD patients before they were diagnosed with lung cancer. This high incidence of ILD seemed to be because many of the patients with ILD were referred to us from other hospitals but followed-up in our hospital. Six of these 9 patients (66.7\%) had LD, whereas only 5 of the remaining $19(26.3 \%)$ had $\mathrm{LD}(P=.095$ by Fisher's exact test). In patients with both ILD and lung cancer, 21 (75.0\%) had idiopathic interstitial pneumonia (IIP) (10 IPF and 11 non-IPF), 6 (21.4\%) had ILD associated with CVD and one (3.6\%) had ILD associated with sarcoidosis. Two patients were on corticosteroid for ILD at baseline when they were diagnosed with SCLC. Pulmonary function tests data were available in 23 of 28 patients with ILD (82.1\%) and 68 of 94 patients without ILD (72.3\%) ( $P=.34$ by Fisher's exact test).

A comparison of patients with and without preexisting ILD is summarized in Table 2. There were no significant differences between the two groups in age $\left(P=.16\right.$ by $\chi^{2}$ test), gender $\left(P=.83\right.$ by $\chi^{2}$ test), smoking status $\left(P=.35\right.$ by $\chi^{2}$ test), ECOG PS ( $P=1.00$ by Fisher's exact test), or stage ( $P$ $=.61$ by $\chi^{2}$ test). Although there were no significant differences in obstructive disorders (forced expiratory volume in one second/forced vital capacity <70\%) ( $P=.43$ by $\chi^{2}$ test), restrictive disorders (percent vital capacity; \% VC $<80 \%$ ) were present significantly more frequently in patients with preexisting ILD ( $P=.042$ by Fisher's exact test). Significantly more patients without preexisting ILD received thoracic radiotherapy combined with chemotherapy $(P=.0038$ by Fisher's exact test). Considering LD-SCLC, only 2 of 11 patients with preexisting ILD received thoracic radiotherapy together with chemotherapy, compared to 33 of 42 without preexisting ILD $(P=.0004$ by Fisher's exact test; $18.2 \%$ vs. $78.6 \%$ ). The response rate of patients without preexisting ILD to initial treatment tended to be higher than that of patients with preexisting ILD, but this difference did not achieve significance ( $P=.063$ by $\chi^{2}$ test; $85 / 93$ vs. $22 / 28$ ). Similarly, for patients who received chemotherapy alone, there were no significant differences in response rates $\left(P=.26\right.$ by $\chi^{2}$ test; $52 / 60$ vs. 20/26). Sixty of 91 patients without preexisting ILD (65.9\%) and 12 of 27 patients with preexisting ILD (44.4\%) received subsequent chemotherapy ( $P=.044$ by $\chi^{2}$ test).

When patients were suspected of having pneumonitis, including AE-ILD, associated with chemotherapy, all received HRCT, sputum culture tests, blood culture tests, and serologic tests (for beta-glucan, cytomegalovirus antigen, tumor markers, brain natriuretic peptide, KL-6, and surfactant protein-D). In addition, 2 patients underwent bronchoscopy; however, this was not possible in the remaining patients due to hypoxia. From these examinations, 10 patients were diagnosed with 
pneumonitis associated with chemotherapy, and 2 with preexisting ILD were diagnosed with opportunistic infections (pneumocystis jirovecii pneumonia and aspergillus pneumonia). Pneumonitis, including AE-ILD, associated with chemotherapy, occurred significantly more frequently in patients with preexisting ILD $(P=.0001$ by Fisher's exact test). Pneumonitis associated with 1 st-line chemotherapy was significantly increased in patients with preexisting ILD $(P=.0024$ by Fisher's exact test; $5 / 28$ vs. 1/94). Five of these 6 patients received combination chemotherapy with platinum and etoposide and one received platinum and irinotecan. Similarly, pneumonitis associated with subsequent chemotherapy also occurred significantly more often in patients with preexisting ILD ( $P=.013$ by Fisher's exact test; $3 / 12$ vs. $1 / 60$ ). Three of these 4 patients received amrubicin and one received paclitaxel. Preexisting ILD was significantly associated with pneumonitis in most chemotherapy regimens (Table 3). Patients with IPF had AE-ILD complications as frequently as patients with ILD other than IPF $(P=1.00$ by Fisher's exact test; $3 / 10$ vs. 5/18). None of the 10 patients was on corticosteroids at baseline and all received high-dose corticosteroid as well as treatment with broad spectrum antibiotics until the possibility of infection was ruled out. Corticosteroid therapy was tapered. However, hHalf of the 10 patients died from pneumonitis. Only 2 of the 10 had mild pneumonitis (grade 2) and could receive subsequent treatment.

Median OS of all patients $(\mathrm{n}=122)$ was 15.5 months, but patients without preexisting ILD had a significantly longer OS than those with preexisting ILD ( $P=.0010$ by log-rank test; 17.8 months vs. 10.7 months) (Fig. 1). The results of univariate and multivariate analyses for OS are summarized in Table 4A. Based on the multivariate analysis, age ( $<75 / \geq 75$ years), gender (female/male), smoking status ( $<40 />40$ pack-years), ECOG PS ( 0 or $1 / 2$ or 3 ), stage (LD/ED), preexisting ILD (no/yes), ECOG PS of 0 or 1 (HR, 0.19; 95\% CI, $0.10-0.37 ; P<.0001$ ), LD (HR, 0.42; 95\% CI, $0.23-0.73$; $P=.0017$ ), and no preexisting ILD (HR, 0.36; 95\% CI, $0.19-0.69 ; P=.0027)$ were all significantly associated with longer OS (Table 4A). Stage (LD/ED) was strongly associated with initial treatment decisions (thoracic radiotherapy with chemotherapy/chemotherapy alone). Therefore, this variable was not included in this analysis. In addition, the variables of pulmonary function tests were also not included since they had some missing values.

Next, we focused on patients receiving chemotherapy alone $(n=87)$. Median PFS on 1st-line chemotherapy was 5.3 months, with no significant differences between patients with or without preexisting ILD ( $P=.089$ by log-rank test; 4.4 months vs. 5.4 months) (Fig. 2). In contrast, median OS of the 87 chemotherapy patients was 12.9 months but when stratified according to the presence of ILD it was significantly longer if there was no ILD ( $P=.027$ by log-rank test; 13.1 months vs. 9.9 months) (Fig. 3). The results of univariate and multivariate analyses for OS are summarized in Table 4B. Based on the multivariate analysis, age ( $<75 / \geq 75$ years), gender (female/male), smoking status ( $<40 / \geq 40$ pack-years), ECOG PS (0 or 1/2 or 3), stage (LD/ED), preexisting ILD (no/yes), ECOG PS 
of 0 or 1 (HR, 0.18; 95\% CI, $0.095-0.36 ; P<.0001)$ and no preexisting ILD (HR, 0.44; 95\% CI, $0.23-0.87 ; P=.020$ ) were all significantly associated with longer OS (Table 4B). 


\section{Discussion}

Although small and retrospective, this study showed that preexisting ILD was significantly associated with worse prognosis in patients with SCLC. To the best of our knowledge, this is the first report that preexisting ILD is an independent prognostic factor for survival in SCLC.

Considering only patients who received chemotherapy alone (no radiotherapy), this study documented no significant differences in response rate to 1st-line chemotherapy, or PFS, between patients with or without preexisting ILD. This indicates that patients with SCLC together with ILD might benefit from chemotherapy considering that when left untreated they rarely survive longer than a few months. ${ }^{3}$ In spite of this finding, this study showed that preexisting ILD is an independent prognostic factor for survival. One reason seemed to be that patients with preexisting ILD rarely received thoracic radiotherapy combined with chemotherapy even if the primary stage was LD. Some studies have shown that preexisting ILD is one of the risk factors for severe radiation pneumonitis and treatment-related death. ${ }^{18,19}$ Therefore, most patients with preexisting ILD do not receive thoracic radiotherapy in our hospital even if they have only LD. Focusing on these patients who only received chemotherapy, however, preexisting ILD also became an independent prognostic factor for survival. This seemed to be because approximate $20 \%$ of patients with preexisting ILD had AE-ILD as a complication and subsequent treatment was limited. In a Japanese case-controlled study, preexisting ILD was reported to be an independent risk factor for developing pneumonitis. ${ }^{20}$ In our study, although the number of patients was small, pneumonitis associated with chemotherapy also occurred significantly more frequently in patients with preexisting ILD. In addition, half of them died from pneumonitis and only 2 of these 10 patients received any subsequent treatment. This high mortality rate was similar to that seen in previous studies. ${ }^{12-15,20}$ In addition, opportunistic infections during immunosuppression and lower \%VC of patients with preexisting ILD can also be associated with poor prognosis.

In some patients, it can be difficult to differentiate pneumonitis, including AE-ILD, associated with chemotherapy from other diseases. For differential diagnosis of pneumonitis, there are various infectious diseases, congestive heart failure, thromboembolism, and progressive lymphangitic spread of the carcinoma that need to be excluded. Therefore, we routinely performed detailed examinations including HRCT, which is a useful modality for the diagnosis of pneumonitis. Nevertheless, the exact incidence of pneumonitis may be overdiagnosed, because radiological changes cannot specifically identify or diagnose histopathological changes.

Combination chemotherapy with platinum and etoposide is considered to be the standard 1st-line chemotherapy regimen for SCLC. ${ }^{21}$ Recently, combination chemotherapy with platinum and irinotecan has been also proposed as a standard 1st-line chemotherapy regimen. ${ }^{22,23}$ Pulmonary toxicity, however, has been reported in patients given irinotecan. ${ }^{24,25}$ Therefore, Minegishi et al. suggested that combination chemotherapy with platinum and etoposide could be a candidate regimen 
for treatment of patients with SCLC together with ILD. In our study, most patients with preexisting ILD did receive platinum plus etoposide (25/28, 89.3\%), and 4 of these suffered AE-ILD as a complication (16.0\%). This rate is similar to that reported in previous studies. ${ }^{12-15}$ Although patients with SCLC together with ILD may benefit from chemotherapy regimens such as platinum plus etoposide, chemotherapy should be administered carefully considering this high AE-ILD rate.

Several clinical trials indicated that amrubicin exhibits significant activity against SCLC and can be effective as a 2 nd-line regimen. ${ }^{26,27}$ However, severe pneumonitis associated with amrubicin has been reported and Yoh et al. recommended not administering this drug to patients with SCLC and ILD. ${ }^{28}$ Our study also noted a high rate of AE-ILD in patients with preexisting ILD who received amrubicin $(2 / 9,22.2 \%)$, similar to a previous report. ${ }^{28}$ Considering this high AE-ILD rate, it is controversial whether patients with SCLC and ILD benefit from amrubicin as a subsequent chemotherapy. Therefore, amrubicin should be administered with caution to selected patients with SCLC and ILD.

Both etoposide and amrubicin are potent inhibitors of topoisomerase II, but one induced pneumonitis and the other not. The precise reason for this is unclear. Etoposide is a rather old drug evaluated during a period without HRCT; therefore, pneumonitis associated with etoposide, including subclinical, could be more frequent than beleived. We suggest another hypothesis; gefitinib and erlotinib are both epidermal growth factor receptor tyrosine kinase inhibitors but several reports have demonstrated successful treatment with erlotinib after gefitinib-induced pneumonitis. ${ }^{29-31}$ Therefore, we speculate that differences in the chemical structure can influence lung toxicity even if the drugs have the same mechanism of action. 


\section{Conclusion}

Although this was a small retrospective analysis, we show here that patients with SCLC together with ILD might benefit from chemotherapy but that preexisting ILD is an independent prognostic factor for survival. Patients with preexisting ILD rarely received thoracic radiotherapy and approximately $20 \%$ of such patients suffered from AE-ILD as a complication, which seems to be associated with poor prognosis. In order to confirm these findings, large prospective studies should be performed. 


\section{Clinical practice points}

Acute exacerbation (AE) of interstitial lung disease (ILD) is a serious complication in patients with lung cancer and simultaneous ILD. Some previous reports suggest that patients with ILD and small cell lung cancer (SCLC) might benefit from chemotherapy, but pneumonitis associated with chemotherapy including AE-ILD is significantly increased in patients with ILD. However, the influence of ILD on prognosis is unclear. In this study, we retrospectively investigated patients with SCLC with or without ILD. We show here that patients with SCLC together with ILD might benefit from chemotherapy but that preexisting ILD is an independent prognostic factor for survival. Patients with preexisting ILD rarely received thoracic radiotherapy and approximately $20 \%$ of such patients suffered from AE-ILD as a complication, which seems to be associated with poor prognosis. From these findings, patients with SCLC and ILD should be received chemotherapy, but caution must be exercised. 


\section{References}

1. Jackman DM, Johnson BE. Small-cell lung cancer. Lancet 2005; 366: 1385-96.

2. Puglisi M, Dolly S, Faria A, et al. Treatment options for small cell lung cancer - do we have more choice? Br J Cancer 2010; 102: 629-38.

3. Demedts IK, Vermaelen KY, van Meerbeeck JP. Treatment of extensive-stage small cell lung carcinoma: current status and future prospects. Eur Respir J 2010; 35: 202-15.

4. Hubbard R, Venn A, Lewis S, et al. Lung cancer and cryptogenic fibrosing alveolitis. A population-based cohort study. Am J Respir Crit Care Med 2000; 161: 5-8.

5. Park J, Kim DS, Shim TS, et al. Lung cancer in patients with idiopathic pulmonary fibrosis. Eur Respir J 2001; 17; 1216-9.

6. Harris JM, Johnston ID, Rudd R, et al. Cryptogenic fibrosing alveolitis and lung cancer: the BTS study. Thorax 2010; 65: 70-6.

7. American Thoracic Society; European Respiratory Society. American Thoracic Society/European Respiratory Society International Multidisciplinary Consensus Classification of the Idiopathic Interstitial Pneumonias. This joint statement of the American Thoracic Society (ATS), and the European Respiratory Society (ERS) was adopted by the ATS board of directors, June 2001 and by the ERS Executive Committee, June 2001. Am J Respir Crit Care Med 2002; 165: 277-304.

8. Collard HR, Moore BB, Flaherty KR, et al; Idiopathic Pulmonary Fibrosis Clinical Research Network Investigators. Acute exacerbations of idiopathic pulmonary fibrosis. Am J Respir Crit Care Med 2007; 176: 636-43.

9. Kondoh Y, Taniguchi H, Kawabata Y, et al. Acute exacerbation in idiopathic pulmonary fibrosis. Analysis of clinical and pathologic findings in three cases. Chest 1993; 103: 1808-12.

10. Hyzy R, Huang S, Myers J, et al. Acute exacerbation of idiopathic pulmonary fibrosis. Chest 2007; 132: 1652-8.

11. Papanikolaou IC, Drakopanagiotakis F, Polychronopoulos VS. Acute exacerbations of interstitial lung diseases. Curr Opin Pulm Med 2010; 16: 480-6.

12. Minegishi Y, Takenaka K, Mizutani H, et al. Exacerbation of idiopathic interstitial pneumonias associated with lung cancer therapy. Intern Med 2009; 48: 665-72.

13. Shukuya T, Ishiwata T, Hara M, et al. Carboplatin plus weekly paclitaxel treatment in non-small cell lung cancer patients with interstitial lung disease. Anticancer Res 2010; 30: 4357-61.

14. Minegishi Y, Kuribayashi H, Kitamura K, et al. The Feasibility Study of Carboplatin Plus Etoposide for Advanced Small Cell Lung Cancer with Idiopathic Interstitial Pneumonias. $J$ Thorac Oncol 2011; 6: 801-7. 
15. Minegishi Y, Sudoh J, Kuribayasi H, et al. The safety and efficacy of weekly paclitaxel in combination with carboplatin for advanced non-small cell lung cancer with idiopathic interstitial pneumonias. Lung Cancer 2011; 71: 70-4.

16. Therasse P, Arbuck SG, Eisenhauer EA, et al. New guidelines to evaluate the response to treatment in solid tumors. European Organization for Research and Treatment of Cancer, National Cancer Institute of the United States, National Cancer Institute of Canada. J Natl Cancer Inst 2000; 92: 205-16.

17. Akira M, Hamada $\mathrm{H}$, Sakatani M, et al. CT findings during phase of accelerated deterioration in patients with idiopathic pulmonary fibrosis. AJR Am J Roentgenol 1997; 168: 79-83.

18. Makimoto T, Tsuchiya S, Hayakawa K, et al. Risk factors for severe radiation pneumonitis in lung cancer. Jpn J Clin Oncol 1999; 29: 192-7.

19. Ohe Y, Yamamoto S, Suzuki K, et al. Risk factors of treatment-related death in chemotherapy and thoracic radiotherapy for lung cancer. Eur J Cancer 2001; 37: 54-63.

20. Kudoh S, Kato H, Nishiwaki Y, et al. Interstitial lung disease in Japanese patients with lung cancer: a cohort and nested case-control study. Am J Respir Crit Care Med 2008; 177: 1348-57.

21. Sundstrøm S, Bremnes RM, Kaasa S, et al; Norwegian Lung Cancer Study Group. Cisplatin and etoposide regimen is superior to cyclophosphamide, epirubicin, and vincristine regimen in small-cell lung cancer: results from a randomized phase III trial with 5 years' follow-up. J Clin Oncol 2002; 20: 4665-72.

22. Noda K, Nishiwaki Y, Kawahara M, et al; Japan Clinical Oncology Group. Irinotecan plus cisplatin compared with etoposide plus cisplatin for extensive small-cell lung cancer. $N$ Engl $J$ Med 2002; 346: 85-91.

23. Lima JP, dos Santos LV, Sasse EC, et al. Camptothecins compared with etoposide in combination with platinum analog in extensive stage small cell lung cancer: systematic review with meta-analysis. J Thorac Oncol 2010; 5: 1986-97.

24. Madarnas Y, Webster P, Shorter AM, et al. Irinotecan-associated pulmonary toxicity. Anticancer Drugs 2000; 11: 709-13.

25. Michielin O, Udry E, Périard D, et al. Irinotecan-induced interstitial pneumonia. Lancet Oncol 2004; 5: 322-4.

26. Onoda S, Masuda N, Seto T, et al; Thoracic Oncology Research Group Study 0301. Phase II trial of amrubicin for treatment of refractory or relapsed small-cell lung cancer: Thoracic Oncology Research Group Study 0301. J Clin Oncol 2006; 24: 5448-53.

27. Inoue A, Sugawara S, Yamazaki K, et al. Randomized phase II trial comparing amrubicin with topotecan in patients with previously treated small-cell lung cancer: North Japan Lung Cancer Study Group Trial 0402. J Clin Oncol 2008; 26: 5401-6. 
28. Yoh K, Kenmotsu H, Yamaguchi Y, et al. Severe interstitial lung disease associated with amrubicin treatment. J Thorac Oncol 2010; 5: 1435-8.

29. Fukui T, Otani S, Hataishi R, et al. Successful rechallenge with erlotinib in a patient with EGFR-mutant lung adenocarcinoma who developed gefitinib-related interstitial lung disease. Cancer Chemother Pharmacol 2010; 65: 803-6.

30. Takeda M, Okamoto I, Makimura C, et al. Successful treatment with erlotinib after gefitinib-induced severe interstitial lung disease. J Thorac Oncol 2010; 5: 1103-4.

28.31. Chang SC, Chamg CY, Chen CY, et al. Successful elotinib rechallenge after gefitinib-induced acute interstitial pneumonia. J Thorac Oncol 2010; 5: 1105-6. 


\section{Figure legends}

Figure 1.

Kaplan-Meier curves of overall survival (OS) for patients with preexisting interstitial lung disease (ILD) vs. patients without preexisting ILD. The median OS of patients without preexisting ILD was significantly longer than that of patients with preexisting ILD $(P=.0010$ by log-rank test; 17.8 months vs. 10.7 months).

\section{Figure 2.}

Kaplan-Meier curves of progression-free survival (PFS) for patients with preexisting interstitial lung disease (ILD) vs. patients without preexisting ILD. Patients received chemotherapy alone ( $\mathrm{n}=87$ ). The median PFS of patients without preexisting ILD was preferentially but not significantly longer than that of patients with preexisting ILD ( $P=.089$ by log-rank test; 4.4 months vs. 5.4 months).

\section{Figure 3.}

Kaplan-Meier curves of overall survival (OS) for patients with preexisting interstitial lung disease (ILD) vs. patients without preexisting ILD. Patients received chemotherapy alone $(n=87)$. The median OS of patients without preexisting ILD was significantly longer than that of patients with preexisting ILD ( $P=.027$ by log-rank test; 13.1 months vs. 9.9 months). 
Table 1. Patients characteristics $(n=122)$.

\begin{tabular}{|c|c|c|}
\hline \multirow{2}{*}{$\begin{array}{l}\text { Patients characteristics } \\
\text { Age (years) }\end{array}$} & Number of patients & \multirow[t]{2}{*}{$\%$} \\
\hline & \multirow{2}{*}{\multicolumn{2}{|c|}{$33-85$}} \\
\hline Range & & \\
\hline Median & \multicolumn{2}{|c|}{69} \\
\hline Mean \pm SD & \multicolumn{2}{|c|}{$67.8 \pm 9.2$} \\
\hline \multicolumn{3}{|l|}{ Gender } \\
\hline Male & 94 & 77.0 \\
\hline Female & 28 & 23.0 \\
\hline \multicolumn{3}{|l|}{ Smoking status } \\
\hline$<40$ pack-years & 95 & 77.9 \\
\hline$\geq 40$ pack-years & 27 & 22.1 \\
\hline \multicolumn{3}{|l|}{ ECOG PS } \\
\hline 0 & 49 & 40.2 \\
\hline 1 & 50 & 41.0 \\
\hline 2 & 16 & 13.1 \\
\hline 3 & 7 & 5.7 \\
\hline \multicolumn{3}{|l|}{ Stage } \\
\hline LD & 53 & 43.4 \\
\hline ED & 69 & 56.6 \\
\hline \multicolumn{3}{|l|}{ ILD } \\
\hline No & 94 & 77.1 \\
\hline IIP & 21 & 17.2 \\
\hline ILD associated with CVD & 6 & 4.9 \\
\hline ILD associated with sarcoidosis & 1 & 0.8 \\
\hline \multicolumn{3}{|l|}{$\% \mathrm{VC}(\%)$} \\
\hline$<80 \%$ & 9 & 7.4 \\
\hline$>80 \%$ & $\underline{82}$ & $\overline{67.2}$ \\
\hline$\overline{\text { Not evaluated }}$ & $\overline{31}$ & 25.4 \\
\hline \multicolumn{3}{|l|}{ FEV 1 /FVC } \\
\hline$<70 \%$ & 42 & 34.4 \\
\hline$>70 \%$ & $\overline{49}$ & 40.2 \\
\hline Not evaluated & $\overline{31}$ & $\overline{25.4}$ \\
\hline \multicolumn{3}{|l|}{ Initial treatment } \\
\hline Thoracic radiotherapy with chemotherapy & 37 & 30.3 \\
\hline Platinum plus etoposide & 53 & 43.5 \\
\hline Platinum plus irinotecan & 32 & 26.2 \\
\hline \multicolumn{3}{|l|}{ Response } \\
\hline Complete response & 23 & 18.9 \\
\hline Partial response & 84 & 68.9 \\
\hline Stable disease & 9 & 7.4 \\
\hline Progressive disease & 5 & 4.1 \\
\hline Not evaluated & 1 & 0.8 \\
\hline
\end{tabular}

Abbreviations; SD = standard deviation; ECOG PS = Eastern Cooperative Oncology Group Performance Status; LD = limited disease; $\mathrm{ED}=$ extensive disease; ILD = interstitial lung

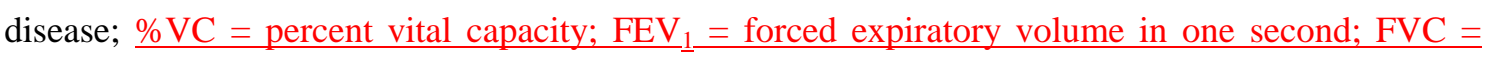
forced vital capacity; IIP = idiopathic interstitial pneumonia; CVD = collagen-vascular disease. 
Table 2. Comparison of patients with or without preexisting ILD $(n=122)$.

\begin{tabular}{|c|c|c|c|}
\hline Patients characteristics & $\begin{array}{c}\text { ILD (+) } \\
(\mathbf{n}=\mathbf{2 8})\end{array}$ & $\begin{array}{l}\text { ILD (-) } \\
(\mathbf{n}=94)\end{array}$ & $P$-value \\
\hline \multicolumn{4}{|l|}{ Age } \\
\hline$<75$ years & 17 & 70 & \multirow{2}{*}{.16} \\
\hline$\geq 75$ years & 11 & 24 & \\
\hline \multicolumn{4}{|l|}{ Gender } \\
\hline Male & 22 & 72 & \multirow{2}{*}{.83} \\
\hline Female & 6 & 22 & \\
\hline \multicolumn{4}{|l|}{ Smoking status } \\
\hline$<40$ pack-years & 8 & 19 & \multirow{2}{*}{.35} \\
\hline$\geq 40$ pack-years & 20 & 75 & \\
\hline \multicolumn{4}{|l|}{ ECOG PS } \\
\hline 0 or 1 & 23 & 76 & \multirow{2}{*}{1.00} \\
\hline 2 or 3 & 5 & 18 & \\
\hline \multicolumn{4}{|l|}{ Stage } \\
\hline $\mathrm{LD}$ & 11 & 42 & \multirow{2}{*}{.61} \\
\hline $\mathrm{ED}$ & 17 & 52 & \\
\hline \multicolumn{4}{|l|}{$\% \mathrm{VC}$} \\
\hline$<80 \%$ & $\underline{5}$ & $\underline{4}$ & \multirow{3}{*}{$.042 *$} \\
\hline$>80 \%$ & $\underline{18}$ & $\underline{64}$ & \\
\hline Not evaluated & $\overline{5}$ & $\overline{26}$ & \\
\hline \multicolumn{4}{|l|}{ FEV $_{1} /$ FVC } \\
\hline$<70 \%$ & $\underline{9}$ & $\underline{35}$ & \multirow{3}{*}{.43} \\
\hline$>70 \%$ & 14 & 33 & \\
\hline Not evaluated & $\underline{5}$ & $\underline{26}$ & \\
\hline \multicolumn{4}{|l|}{ Initial treatment } \\
\hline Thoracic radiotherapy with chemotherapy & 2 & 33 & \multirow{2}{*}{$.0038^{*}$} \\
\hline Chemotherapy alone & 26 & 61 & \\
\hline \multicolumn{4}{|l|}{ First-line chemotherapy regimen } \\
\hline Platinum with etoposide & 25 & 65 & \multirow{2}{*}{$.048^{*}$} \\
\hline Platinum with irinotecan & 3 & 29 & \\
\hline \multicolumn{4}{|l|}{ Response to initial treatment } \\
\hline $\mathrm{CR}$ or PR & 22 & 85 & \multirow{3}{*}{.063} \\
\hline $\mathrm{SD}$ or $\mathrm{PD}$ & 6 & 8 & \\
\hline Not evaluated & 0 & 1 & \\
\hline \multicolumn{4}{|l|}{ Subsequent chemotherapy } \\
\hline Yes & 12 & 60 & \multirow{3}{*}{$.044 *$} \\
\hline No & 15 & 31 & \\
\hline Unknown & 1 & 3 & \\
\hline \multicolumn{4}{|l|}{ Pneumonitis associated with chemotherapy } \\
\hline Yes & 8 & 2 & \multirow{2}{*}{$.0001 *$} \\
\hline No & 20 & 92 & \\
\hline
\end{tabular}

* Statistically significant with the $\chi^{2}$ test or Fisher'sexacttest; $P<.05$.

Abbreviations; ILD = interstitial lung disease; ECOG PS = Eastern Cooperative Oncology Group Performance Status; $\mathrm{LD}=$ limited disease $; \mathrm{ED}=$ extensive disease; $\% \mathrm{VC}=$ percent vital capacity; $\underline{\mathrm{FEV}}_{1}=$ forced expiratory volume in one second; $\mathrm{FVC}=$ forced vital capacity; $\mathrm{CR}=$ complete response $; \mathrm{PR}=$ partial response $; \mathrm{SD}=$ stable disease $; \mathrm{PD}=$ progressive disease . 
Table 3. Pneumonitis associated with the different chemotherapy regimens.

\begin{tabular}{lccc}
\hline Chemotherapy & $\begin{array}{c}\text { ILD (+) } \\
(\mathbf{n = 2 8 )}\end{array}$ & $\begin{array}{c}\text { ILD (-) } \\
(\mathbf{n}=94)\end{array}$ & P-value \\
\hline Total & $8 / 28(28.6 \%)$ & $2 / 94(2.1 \%)$ & $.0001^{*}$ \\
First-line chemotherapy & $5 / 28(17.9 \%)$ & $1 / 94(1.1 \%)$ & $.0024^{*}$ \\
Platinum plus etoposide & $4 / 25(16.0 \%)$ & $1 / 65(1.5 \%)$ & $.020^{*}$ \\
Platinum plus irinotecan & $1 / 3(33.3 \%)$ & $0 / 29(0 \%)$ & .094 \\
Subsequent chemotherapy & $3 / 12(25 \%)$ & $1 / 60(1.7 \%)$ & $.013^{*}$ \\
Amrubicin & $2 / 9(22.2 \%)$ & $1 / 59(1.7 \%)$ & $.045^{*}$ \\
Paclitaxel & $1 / 1(100.0 \%)$ & $0 / 7(0.0 \%)$ & .13 \\
\hline
\end{tabular}

* Statistically significant with the $\chi^{2}$ test or Fisher'sexacttest; $P<.05$

Abbreviations; ILD = interstitial lung disease. 
Table 4. (A) Univariate and multivariate analyses for overall survival in all patients $(\mathrm{n}=122)$. (B) Univariate and multivariate analyses for overall survival in patients who received chemotherapy alone $(n=87)$.

\begin{tabular}{|c|c|c|c|c|c|c|}
\hline & \multicolumn{3}{|c|}{ Univariate analysis } & \multicolumn{3}{|c|}{ Multivariate analysis } \\
\hline & HR & $95 \% \mathrm{CI}$ & $P$-value & HR & $95 \% \mathrm{CI}$ & $P$-value \\
\hline \multicolumn{7}{|l|}{ (A) Variables } \\
\hline Age $(<75 />75$ years $)$ & 0.88 & $0.54-1.51$ & .64 & 0.91 & $0.53-1.62$ & .75 \\
\hline Gender (female/male) & 0.90 & $0.50-1.54$ & .72 & 0.68 & $0.35-1.24$ & .21 \\
\hline Smoking status (<40/>40 pack-years) & 1.25 & $0.69-2.13$ & .45 & 1.28 & $0.68-2.30$ & .43 \\
\hline ECOG PS $(0$ or $1 / 2$ or 3$)$ & 0.20 & $0.11-0.37$ & $<.0001 *$ & 0.19 & $0.10-0.37$ & $<.0001 *$ \\
\hline Stage (LD/ED) & 0.35 & $0.20-0.59$ & $<.0001 *$ & 0.42 & $0.23-0.73$ & $.0017 *$ \\
\hline Preexisting ILD (no/yes) & 0.40 & $0.23-0.72$ & $.0030^{*}$ & 0.36 & $0.19-0.69$ & $.0027 *$ \\
\hline \multicolumn{7}{|l|}{ (B) Variables } \\
\hline Age $(<75 />75$ years $)$ & 0.75 & $0.45-1.31$ & .31 & 0.74 & $0.41-1.37$ & .34 \\
\hline Gender (female/male) & 0.76 & $0.39-1.36$ & .36 & 0.68 & $0.34-1.31$ & .26 \\
\hline Smoking status (<40/ $>40$ pack-years $)$ & 1.08 & $0.59-1.87$ & .79 & 1.16 & $0.61-2.12$ & 63 \\
\hline ECOG PS $(0$ or $1 / 2$ or 3$)$ & 0.26 & $0.14-0.49$ & $<.0001 *$ & 0.18 & $0.095-0.36$ & $<.0001 *$ \\
\hline Stage (LD/ED) & 0.88 & $0.43-1.63$ & .70 & 0.80 & $0.38-1.56$ & .52 \\
\hline Preexisting ILD (no/yes) & 0.53 & $0.30-0.96$ & $.037 *$ & 0.44 & $0.23-0.87$ & $.020 *$ \\
\hline
\end{tabular}

* Statistically significant with logistic regression model analysis; $P<.05$.

Abbreviations; ECOG PS = Eastern Cooperative Oncology Group Performance Status; LD = limited disease; ED = extensive disease; ILD = interstitial lung disease $; \mathrm{HR}=$ hazard ratio $; \mathrm{CI}=$ confidence interval 


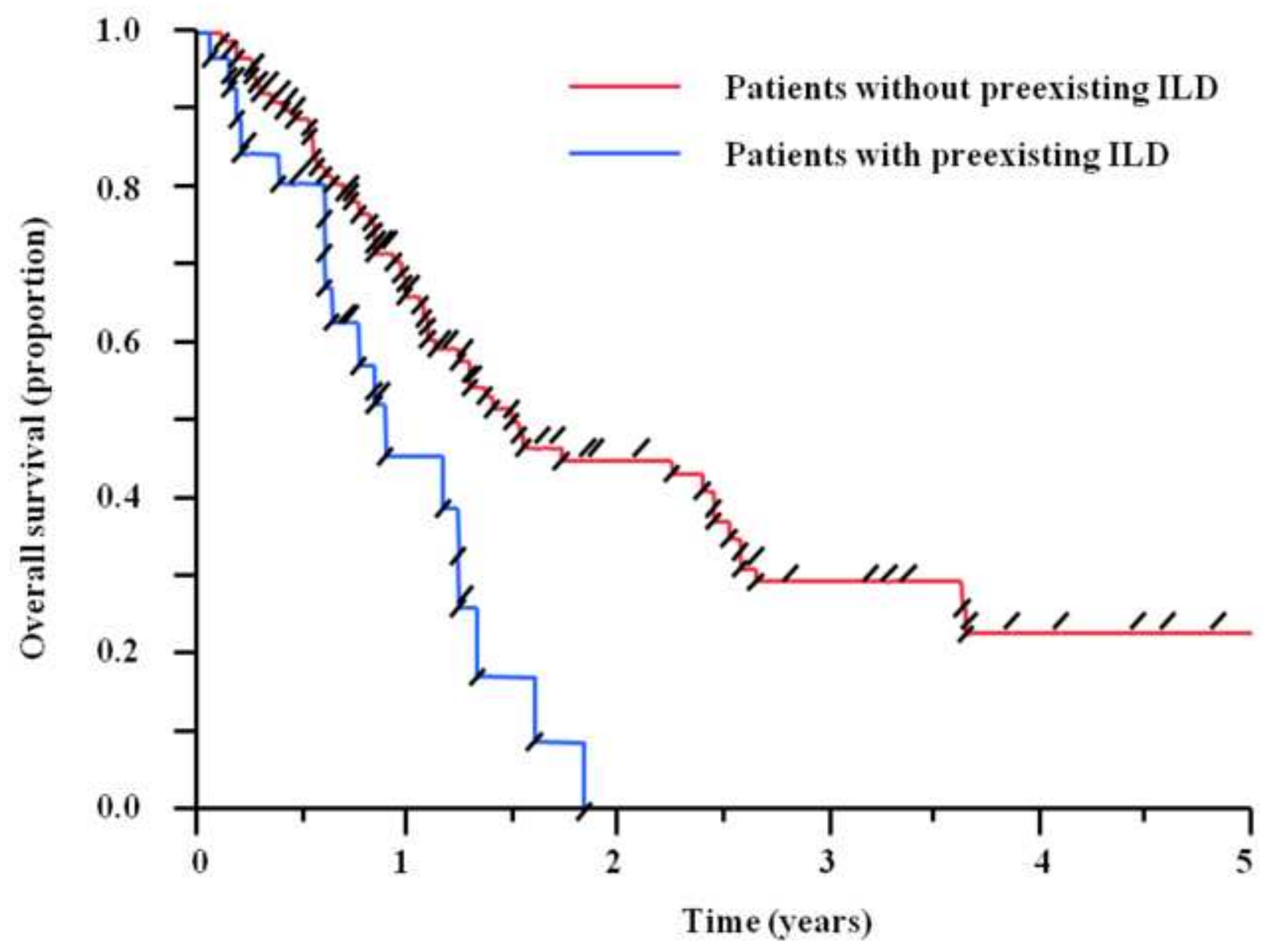




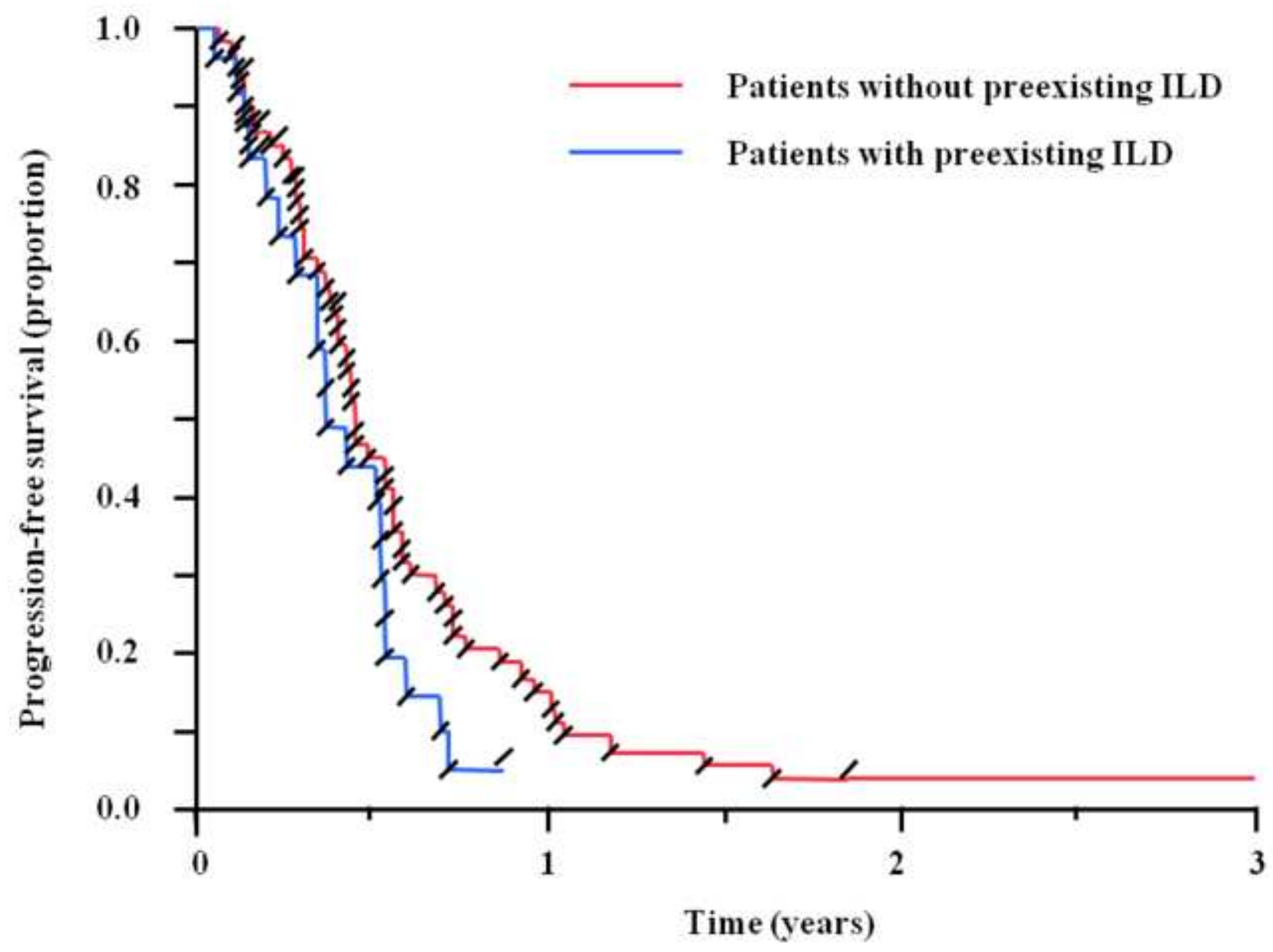




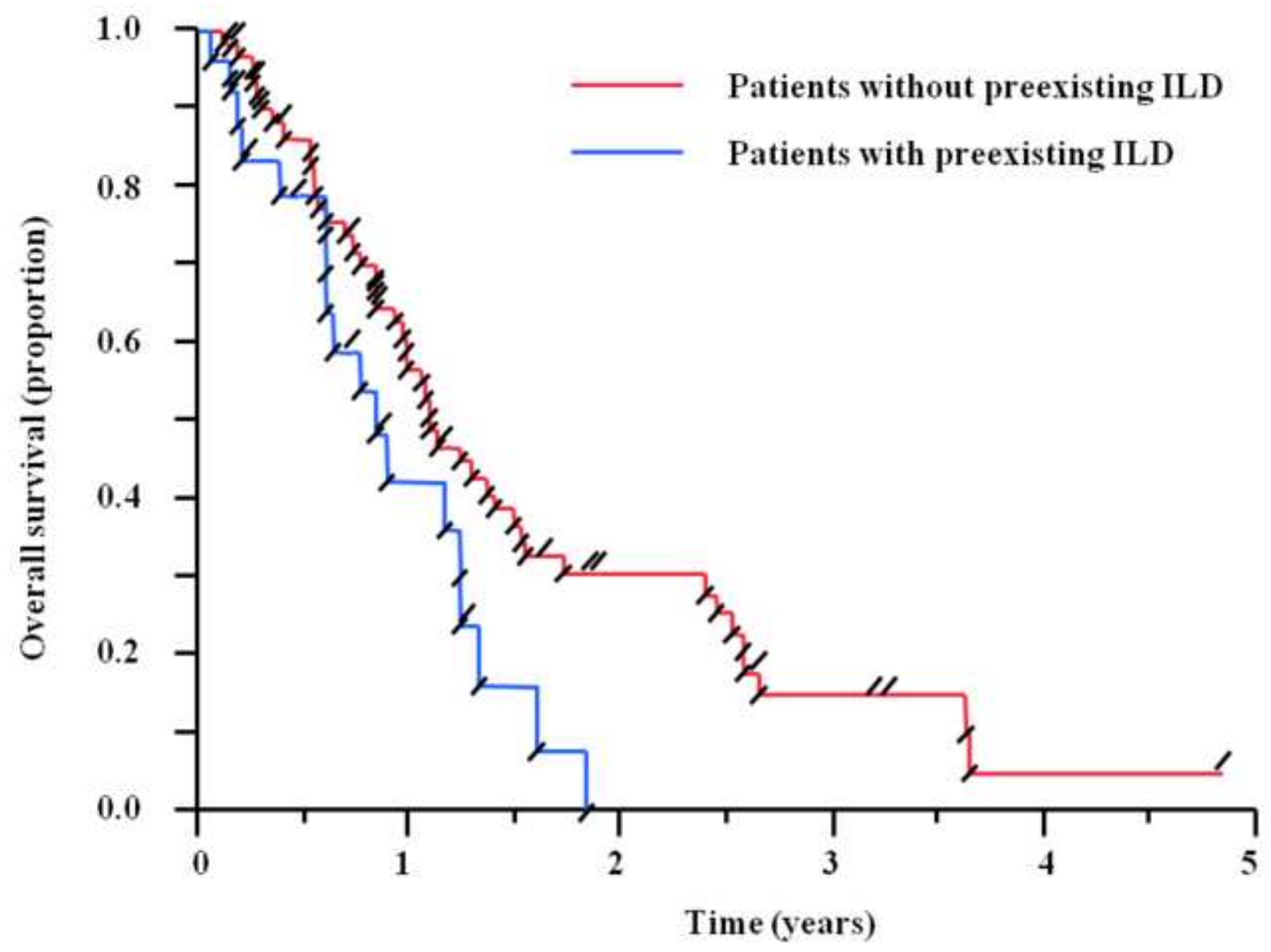

